\title{
Psychometric properties of the Geriatric Anxiety Inventory (GAI) and its short-form (GAI-SF) in a clinical and non-clinical sample of older adults
}

\author{
Carly Johnco, Ashleigh Knight, Dusanka Tadic and Viviana M. Wuthrich \\ Centre for Emotional Health, Department of Psychology, Macquarie University, Sydney, New South Wales, Australia
}

ABSTRACT

Background: The Geriatric Anxiety Inventory is a 20 -item geriatric-specific measure of anxiety severity. While studies suggest good internal consistency and convergent validity, divergent validity from measures of depression are weak. Clinical cutoffs have been developed that vary across studies due to the small clinical samples used. A six-item short form (GAI-SF) has been developed, and while this scale is promising, the research assessing the psychometrics of this scale is limited.

Methods: This study examined the psychometric properties of GAI and GAI-SF in a large sample of 197 clinical geriatric participants with a comorbid anxiety and unipolar mood disorder, and a non-clinical control sample $(N=59)$.

Results: The internal consistency and convergent validity with other measures of anxiety was adequate for GAI and GAI-SF. Divergent validity from depressive symptoms was good in the clinical sample but weak in the total and non-clinical samples. Divergent validity from cognitive functioning was good in all samples. The one-factor structure was replicated for both measures. Receiver Operating Characteristic analyses indicated that the GAI is more accurate at identifying clinical status than the GAI-SF, although the sensitivity and specificity for the recommended cutoffs was adequate for both measures.

Conclusions: Both GAI and GAI-SF show good psychometric properties for identifying geriatric anxiety. The GAI-SF may be a useful alternative screening measure for identifying anxiety in older adults.

Key words: anxiety disorders, anxiety, questionnaires, geriatric, Geriatric Anxiety Inventory, older adult

\section{Introduction}

The Geriatric Anxiety Inventory (GAI) was developed as a brief dimensional measure of anxiety symptom severity specifically for use with older people (Pachana et al., 2007). The initial study found adequate psychometric properties in a large sample of normal older adults and a smaller sample of psychogeriatric patients (Pachana et al., 2007). Since this initial study, there has been rapid growth in the number of studies evaluating the psychometric properties of this measure in a variety of older adult settings, including community samples (Byrne et al., 2010), psychogeriatric samples (Cheung, 2007; Boddice et al., 2008), residential aged care settings (Boddice et al., 2008), and in-home care recipients (Diefenbach

Correspondence should be addressed to: Dr Viviana M. Wuthrich, Centre for Emotional Health, Macquarie University, Sydney, New South Wales, Australia. Phone: +61(2) 9850 4866; Fax: +61(2) 9850 8062. Email: Viviana. Wuthrich@mq.edu.au. Received 25 Apr 2014; revision requested 23 Jun 2014; revised version received 29 Jun 2014; accepted 2 Jul 2014. First published online 11 August 2014 et al., 2009). It has also been validated in health settings, including in patients with Parkinson's disease (Matheson et al., 2012), patients with chronic obstructive pulmonary disease (COPD; Cheung et al., 2012), and in patients with varied levels of cognitive impairment (Boddice et al., 2008; Byrne et al., 2008; Rozzini et al., 2009).

The GAI is one of the first self-report measures of late-life anxiety that adequately addresses many of the notable limitations of using measures developed with a younger sample in an older adult population. The GAI minimizes the emphasis of somatic symptoms which can be confounded with physical health problems in older adults (Pachana et al., 2007). The GAI has a forced choice response format (agree/disagree) and is scored in a single direction which serves to reduce confusion that can present with reverse scored items and the scaled response formats often used with younger adults. Psychometric and methodological literature on selfreport measures given to older adults frequently find that reverse scored items form a separate 
factor, sometimes referred to as a "confusion factor" (Green et al., 1993; Hazlett-Stevens et al., 2004; Pachana et al., 2007; Boddice et al., 2008; Carlson et al., 2011), as these negatively worded items require an ability to recognize and interpret double negatives and use the reverse ends of a rating scale to make an appropriate response. Using this single direction response format, the GAI has demonstrated a single factor (Pachana et al., 2007; Byrne et al., 2010). The GAI refers to emotional functioning over a past week, allowing for symptom tracking on a weekly routine clinical care schedule. All of these facets make the GAI a desirable measure for use with older population.

While the GAI provides many age-appropriate features for ease of administration with older adults, the psychometric information from the surge of validation studies shows generally good convergent validity, but weak divergent validity from depression measures. The GAI's internal consistency is consistently good, ranging from $\mathrm{r}=0.91$ to 0.95 (Pachana et al., 2007; Byrne et al., 2008; Matheson et al., 2012). The few studies that have examined testretest reliability find it to be acceptable $(\mathrm{r}=0.91-$ 0.99; Pachana et al., 2007; Matheson et al., 2012), and the original development study found acceptable inter-rater reliability using audio taped responses (Pachana et al., 2007). Convergent validity is also consistently good, with the GAI correlating well with other self-report measures of anxiety. For example, Deifenbach et al. (2009) found a positive correlation of GAI with a range of anxiety measures, including the Generalized Anxiety Disorder (GAD) Questionnaire for the Diagnostic and Statistical Manual of Mental Disorders, Fourth Edition (DSM-IV), $\mathrm{r}=0.653$ (Newman et al., 2002), the Penn State Worry Questionnaire (PSWQ), $\mathrm{r}=$ 0.794 (Meyer et al., 1990), the abbreviated version of PSWQ (PSWQ-A), $\mathrm{r}=0.795$ (Hopko et al., 2003), and the Beck Anxiety Inventory, $r=0.613$ (Beck et al., 1988). Similarly, Pachana et al. (2007) found a positive correlation between GAI and measures such as the Goldberg Anxiety and Depression Scale - anxiety subscale, $\mathrm{r}=0.57$ (Goldberg et al., 1988), the State-Trait Anxiety Inventory-State, $\mathrm{r}=-0.44$ (Spielberger et al., 1970), Beck Anxiety Inventory, $\mathrm{r}=0.63$, and PSWQ, $\mathrm{r}=$ 0.70 . Convergent validity has also been found using structured clinical interviews, including the Mini-International Neuropsychiatric Interview (MINI; Pachana et al., 2007; Byrne et al., 2010; Cheung et al., 2012; Matheson et al., 2012), Anxiety Disorders Interview Schedule (ADIS-IV; Di Nardo et al., 1994), and Composite International Diagnostic Interview (Boddice et al., 2008).

The GAI is frequently correlated with measures of depression. For example, Andrew and Dulin
(2007), Byrne et al. (2008), and Deifenbach et al. (2009) found moderate to strong correlations with GDS ( $\mathrm{r}=0.62,0.79$, and 0.67 respectively). It is difficult to interpret the meaning of these correlations because some authors describe the correlation with measures of depression as evidence of poor divergent validity (e.g., Diefenbach et al., 2009), while others conclude that due to high comorbidity between anxiety and depressive symptoms among older adults the correlation indicates adequate convergent validity (e.g., Cheung, 2007; Byrne et al., 2008). Other studies note the correlation but do not prefer this as good or bad (e.g., Andrew and Dulin, 2007). The correlation between measures of anxiety and depression is frequently noted in studies with younger adults, perhaps indicating that these measures assess negative affects in general, rather than anxiety and depression specifically (Feldman, 1993; Stulz and Crits-Christoph, 2010).

The original development paper compared scores on GAI with diagnosed GAD on MINI (Rozzini et al., 2009) and suggested a cutoff of $10 / 11$ out of 20 for identifying likely GAD, and a cutoff of $8 / 9$ out of 20 for identifying any anxiety disorder (Pachana et al., 2007). However, this psychogeriatric sample was very small $(N=19)$ and had only eight participants with a diagnosis of GAD, and 11 with a diagnosis of other anxiety disorders. Subsequent studies have suggested similar cutoffs but have generally used small numbers of patients with anxiety disorders. Diefenbach et al. (2009) replicated the original findings suggesting a cutoff of 9 for identifying any anxiety disorder; however, again this was based on a small sample of eight participants with any anxiety disorder diagnosis. In a community sample of females, Byrne et al. (2010) suggested a cutoff of $8 / 9$ to predict GAD on MINI, but again only a small number of the sample met the diagnostic criteria $(N=8 / 253)$. In healthy population, a cutoff of $6 / 7$ was found to be optimum for identifying any anxiety disorder in patients with Parkinson's disease, but again using small number with an anxiety disorder diagnosis $(N=16,28 \%$ of the sample; Matheson et al., 2012). A far lower cutoff of $2 / 3$ was suggested to identify patients with any anxiety disorder in a sample with COPD, with $14(25.5 \%$ of the sample) participants meeting the diagnostic criteria (Cheung et al., 2012). Overall, variability in optimal cutoff scores is likely to be an artefact of varied samples and the small number of patients who met criteria for an anxiety disorder. More research in larger clinical samples is needed.

Recently, a short five-item version of GAI was developed (GAI-SF; Byrne and Pachana, 2011); however, there are currently few studies 
evaluating the psychometric properties of this measure, especially in large samples. The scale was developed in a sample consisting of female participants only, so the applicability to a mixed gender sample has not been established. An optimal cutoff of $2 / 3$ was suggested to identify females with any anxiety disorder; however again, only a small number of participants in the sample had a diagnosed anxiety disorder $(N=8,3.3 \%$ of the sample; Byrne and Pachana, 2011). This cutoff had $75 \%$ sensitivity, and specificity of $87 \%$, with $86 \%$ of participants correctly classified (Byrne and Pachana, 2011). Recently, Gerolimatos et al. (2013) examined the psychometric properties of GAI and GAI-SF in 75 nursing home residents and found adequate psychometric properties. This study found moderate correlations between GAI, GAI-SF, and GDS-15, and a good divergent validity from measures of adaptive and executive functioning. Results from this study suggested an optimum cutoff score of 9 for GAI ( $100 \%$ sensitivity and $60 \%$ specificity) and a score of 2 for GAISF (100\% sensitivity and $46.2 \%$ specificity) for identifying those with an anxiety disorder diagnosis. Although this study represents one of the first independent studies to evaluate the psychometric properties of GAI-SF, and also the first to examine this in a residential care setting, this, similar to others, consisted of a small sample with a diagnosed anxiety disorder $(N=10)$.

The present study aims to replicate previous findings on psychometric properties of GAI in a large clinical sample of older adults meeting criteria for an anxiety disorder and a non-clinical control sample. This study also aimed to assess the psychometric properties of GAI-SF, given this measure is brief and shows potential for use in screening or epidemiological settings, but has few studies assessing the reliability and validity of this measure. In particular, we were interested in assessing the factor structure, convergent and divergent validity as well and the internal reliability of the short form in comparison with the full version. In addition, this study aims to investigate the sensitivity and specificity of the recommended optimal cutoffs for GAI and GAI-SF to identify those with an anxiety disorder diagnosis in a more robust psychogeriatric sample with comorbid anxiety and depression.

\section{Method}

\section{Participants}

There were a total of 256 community-dwelling participants ( 161 females, age range $=60-88$ years, Mean $(M)=67.51, S D=5.69)$. Participants were included from data collected as part of other studies, two using a non-clinical control sample of community-dwelling older adults and the clinical participants being drawn from two randomized control trials for the treatment of late life anxiety and depression (Johnco et al., 2013; Wuthrich and Rapee, 2013). The non-clinical sample $(N=$ 59 , females $=42$, age range $=60-86$ years, $\mathrm{M}=67.56, \mathrm{SD}=6.20$ ) was recruited from local newspaper advertisements. Participants contacted the researchers and were screened on telephone for clinically significant mental health problems, and those reporting significant mental health problem were excluded. Participants' scores on the selfreport measures of anxiety and depression fell in a normal range.

The clinical sample $(N=197$, females $=119$, age range $=61-88$ years, $M=67.50, S D=$ 5.55) was a treatment-seeking sample recruited through local newspaper advertisements for two randomized controlled trials (RCTs). The Anxiety Disorders Interview Schedule for DSM-IV (ADISIV; Di Nardo et al., 1994) was administered by postgraduate psychology students and registered psychologists under the supervision of a clinical psychologist. All clinical participants recruited for the trial met criteria for both DSM-IV anxiety and unipolar mood disorder, with either being primary. In the clinical sample, $58 \%(N=115)$ had a primary anxiety disorder (35\% GAD, $8.1 \%$ social phobia) and $42 \%(N=82)$ had a primary unipolar mood disorder (26.9\% major depressive disorder, $10.7 \%$ dysthymia). During selection for RCT, participants reporting current self-harm, active suicidal ideation, psychosis, or bipolar disorder were excluded from the study as the clinic was ill-equipped to deal with these acute risks. Demographic information for both samples is provided in Table 1 .

\section{Measures}

Addenbrooke Cognitive Examination - Revised (ACE$\mathrm{R}$; Mioshi et al., 2006): The ACE-R is a screening instrument designed to assess an individual's cognitive level, and has demonstrated good internal consistency and convergent validity in an older adult sample (Mioshi et al., 2006). This measure was administered to participants by a trained postgraduate psychology student. Results suggest that this sample was cognitively intact with only $2.1 \%$ of the sample scoring in the range indicative of cognitive impairment $(<82 / 100 ; M=92.71$, $\mathrm{SD}=4.80)$. This measure consists of five subscales assessing various aspects of cognitive functioning, and the attention/orientation, memory, fluency, language, and visuospatial subscales are combined to provide an overall total cognitive level score. The 
Table 1. Demographic and descriptive information for non-clinical and clinical samples

\begin{tabular}{|c|c|c|c|}
\hline & $\begin{array}{l}\text { NON-CLINICAL } \\
(N=59)(\%)\end{array}$ & $\begin{array}{l}\text { CLINICAL } \\
(N=197)(\%)\end{array}$ & $F$ \\
\hline Females & 71.2 & 60.4 & 2.26 \\
\hline Marital status & & & 0.09 \\
\hline Never married & 3.4 & 4.6 & \\
\hline Married & 54.2 & 47.2 & \\
\hline De facto & 3.4 & 3 & \\
\hline Divorced/separated & 20.3 & 32.9 & \\
\hline Widowed & 18.6 & 12.2 & \\
\hline Country of birth & & & 1.97 \\
\hline Australia & 69.5 & 66.0 & \\
\hline UK & 18.6 & 10.6 & \\
\hline Other & 11.9 & 23.4 & \\
\hline Highest level of qualification & & & 1.74 \\
\hline Primary school & 0 & 3.6 & \\
\hline Secondary school & 20.3 & 22.8 & \\
\hline Certificate/diploma & 30.5 & 27.9 & \\
\hline Bachelors degree & 32.2 & 19.3 & \\
\hline Postgraduate degree & 15.3 & 14.7 & \\
\hline Other & 1.7 & 11.7 & \\
\hline Employment status & & & 0.17 \\
\hline Employed full-time & 5.1 & 6.6 & \\
\hline Semi-retired & 35.6 & 20.3 & \\
\hline Retired & 55.9 & 67.0 & \\
\hline Unable to work due to illness/injury & 0 & 2 & \\
\hline Unemployed & 3.4 & 3 & \\
\hline Gross income (\$AUD) & & & 1.57 \\
\hline$<\$ 15,599$ & 16.7 & 22.1 & \\
\hline$\$ 15,600-41,599$ & 37.0 & 46.3 & \\
\hline$\$ 41,600-83,199$ & 33.3 & 21.1 & \\
\hline$\$ 83,200-134,199$ & 13.0 & 6.8 & \\
\hline \multirow[t]{2}{*}{$135,200+$} & 0 & 3.7 & \\
\hline & $M(S D)$ & $M(S D)$ & $\mathrm{t}$ \\
\hline GAI & $0.58(1.32)$ & $11.08(4.86)$ & $-27.17^{*}$ \\
\hline GAI-SF & $0.17(0.62)$ & $3.46(1.48)$ & $-27.78^{*}$ \\
\hline GDS & $2.30(2.83)$ & $17.28(5.53)$ & $-27.20^{*}$ \\
\hline PSWQ-A & $13.49(5.40)$ & $25.15(7.59)$ & $-10.65^{*}$ \\
\hline
\end{tabular}

Note: ${ }^{*} \mathrm{p}<0.05$.

attention/orientation, language, and visuospatial subscales were used to assess divergent validity, given they are unlikely to be influenced by anxiety or depression.

Anxiety Disorders Interview Schedule (Di Nardo et al., 1994): The ADIS is a semi-structured interview for diagnosing anxiety and related disorders according to DSM-IV criteria. Trained postgraduate psychology students, who received regular supervision, administered it. The interview is designed to support clinicians in determining the presence and severity of disorders using a rating scale of $0-8$, where ratings of 4 and above are considered of clinical severity. The ADIS-IV was administered in full to the clinical sample only.

Geriatric Anxiety Inventory (Pachana et al., 2007): The GAI is a 20 -item measure of anxiety symptoms severity developed for older adults. It has been shown to have adequate internal consistency, testretest reliability, and concurrent validity (Pachana et al., 2007). Internal consistency was good for the non-clinical $(\alpha=0.73)$ and clinical samples $(\alpha=$ 0.85 ) in the current sample.

Geriatric Anxiety Inventory - Short Form (Byrne and Pachana, 2011): The GAI-SF is an abbreviated version of GAI comprising only five of the original items. The GAI-SF has good internal consistency, convergent and divergent validity, and was highly correlated with the original GAI (Pachana et al., 2007). In the current study, the internal consistency for the non-clinical sample was adequate $(\alpha=0.71)$ but marginal for the clinical sample $(\alpha=0.58)$. The scores from GAI-SF were extracted in the same manner as Byrne and Pachana (2011) from 
the GAI scores as opposed to the two tests being administered separately.

Geriatric Depression Scale (Yesavage et al., 1983): The GDS is a 30 -item self-report measure aimed to determine the severity of depressive symptoms in older adults. It has high internal consistency, reliability, sensitivity, and specificity (Yesavage et al., 1983; Kieffer and Reese, 2002; Jongenelis et al., 2005). Internal reliability was good for both non-clinical $(\alpha=0.79)$ and clinical samples $(\alpha=$ 0.84 ) in the current study.

Penn State Worry Questionnaire - Abbreviated (Hopko et al., 2003): The PSWQ-A is an abbreviated version of PSWQ (Meyer et al., 1990), which contains eight of the original items and assesses worry severity. The PSWQ-A has been shown to be highly correlated with the original measure, have good internal consistency, and convergent-divergent validity in older adult samples (Hopko et al., 2003). In our sample, internal consistency was good for both non-clinical ( $\alpha=$ $0.82)$ and clinical samples $(\alpha=9.90)$.

\section{Procedure}

The Macquarie University Human Ethics Committee granted ethnic approval for all studies, and participants provided written consent. The clinical participants completed the measures as part of an initial assessment prior to treatment for treatment study. The non-clinical participants completed the measures as part of other studies. Analyses were conducted using SPSS version 17 and STATA version 12. 1 (StataCorp. 2011).

\section{Results}

Group differences on demographic characteristics were examined using one-way ANOVA, and no significant differences were found (see Table 1). Independent sample $\mathrm{t}$-tests were conducted to compare group differences on cognitive (ACE-R total score) and symptom measures (GAI, GAI-SF, GDS, and PSWQ-A) and indicated that the clinical sample scored significantly higher on all symptom measures (see Table 1). Missing data were excluded in all analyses in this paper.

Replicating previous studies, the internal reliability was good for GAI in the total sample ( $\alpha=0.926)$, clinical sample $(\alpha=0.854)$, and nonclinical sample $(\alpha=0.714)$. The internal reliability for GAI-SF was good for the total sample ( $\alpha=$ $0.840)$ and the non-clinical sample $(\alpha=0.718)$, and acceptable for the clinical sample $(\alpha=0.669)$.

There was moderate convergent validity of GAI and GAI-SF with another measure of anxiety (PSWQ-A) in the total sample $(\mathrm{r}=0.787, \mathrm{p}<$
0.001 and $\mathrm{r}=0.793, \mathrm{p}<0.001)$, clinical sample $(\mathrm{r}=0.637, \mathrm{p}<0.001$ and $\mathrm{r}=0.641, \mathrm{p}<0.001$ respectively), and non-clinical sample $(\mathrm{r}=0.603, \mathrm{p}$ $<0.001$ and $\mathrm{r}=0.563, \mathrm{p}<0.001)$. The GAI and GAI-SF were significantly correlated in the total sample $(\mathrm{r}=0.934, \mathrm{p}<0.001)$, clinical sample $(\mathrm{r}=0.865, \mathrm{p}<0.001)$ and non-clinical sample $(\mathrm{r}=0.893, \mathrm{p}<0.001)$.

There was some evidence of divergent validity from a measure of geriatric depression (GDS) in the clinical sample with the GAI showing a weaker correlation with the GDS compared with the PSWQ-A $(\mathrm{r}=0.477, \mathrm{p}=0.001 ; \mathrm{z}=2.539, \mathrm{p}=0.011)$. Similarly, the GAI-SF showed a weaker correlation with GDS compared with PSWQ-A ( $\mathrm{r}=0.372$, $\mathrm{p}=0.001 ; \mathrm{z}=4.092, \mathrm{p}<0.001)$, suggesting good divergent validity in the clinical sample. The correlations between GAI and GDS, and GAISF and GDS, were not significantly different in magnitude in relation to PSWQ-A in the total sample $(\mathrm{r}=0.773, \mathrm{p}=0.001 ; \mathrm{z}=0.52, \mathrm{p}=$ 0.603 and $\mathrm{r}=0.737, \mathrm{p}=0.001 ; \mathrm{z}=2.00$, $\mathrm{p}=0.05$ respectively), suggesting poor divergent validity in the total sample. Similarly, there was no significant difference between these relationships in the non-clinical sample $(\mathrm{r}=0.656, \mathrm{p}<0.001$; $\mathrm{z}=-0.554, \mathrm{p}=0.58$ and $\mathrm{r}=0.520, \mathrm{p}<0.001$; $\mathrm{z}=0.401, \mathrm{p}=0.689$ ), suggesting weak divergent validity from measures of depression. Divergent validity from measures of cognitive functioning was assessed using the attention/orientation, language and visuospatial subscales from the ACE-R, given that these subscales are unlikely to be negatively influenced by anxiety and depression (compared with the fluency and memory subscales that are commonly found to be impaired in those with anxiety and depressive disorders). There was good divergent validity for GAI and GAI-SF from the attention/orientation subscale in total, clinical, and non-clinical samples $(\mathrm{r}=-0.048,0.077$, and 0.052 respectively, and all $\mathrm{p}$-values were non-significant) as well as from the language subscale $(r=-0.063$, 0.066 , and -0.018 respectively, all $\mathrm{p}$-values were non-significant) and visuospatial subscale ( $\mathrm{r}=$ $-0.118,-0.074$, and 0.73 respectively, all $p$-values were non-significant).

A factor analysis using principal axis factoring was conducted for total and clinical samples only due to limited variability in the GAI and GAI-SF scores in the non-clinical sample that prohibited separate analysis. In the clinical sample, the Kaiser-Meyer-Olkin Measure of Sampling Adequacy $=0.833$ suggested that the sample was large enough for analysis, and inter-correlations between items were adequate (Bartlett's Test of Sphericity, $\left.\chi^{2}(190)=1004.286, \mathrm{p}<0.001\right)$. Examination of eigenvalues and scree plot suggest 
Table 2. Component matrix of GAI for total sample

\begin{tabular}{lll}
\hline ITEM & CLINICAL SAMPLE & TOTAL SAMPLE \\
\hdashline$\ldots \ldots \ldots \ldots \ldots \ldots \ldots \ldots \ldots$ \\
Q1 & 0.610 & 0.809 \\
Q2 & 0.370 & 0.542 \\
Q3 & 0.558 & 0.644 \\
Q4 & 0.403 & 0.625 \\
Q5 & 0.586 & 0.715 \\
Q6 & 0.599 & 0.682 \\
Q7 & 0.504 & 0.532 \\
Q8 & 0.518 & 0.712 \\
Q9 & 0.548 & 0.655 \\
Q10 & 0.643 & 0.735 \\
Q11 & 0.500 & 0.734 \\
Q12 & 0.382 & 0.435 \\
Q13 & 0.488 & 0.588 \\
Q14 & 0.447 & 0.571 \\
Q15 & 0.501 & 0.574 \\
Q16 & 0.558 & 0.767 \\
Q17 & 0.557 & 0.660 \\
Q18 & 0.482 & 0.533 \\
Q19 & 0.526 & 0.607 \\
Q20 & 0.528 & 0.721 \\
\hline
\end{tabular}

Table 3. Component matrix of GAI-SF for total sample

\begin{tabular}{llc}
\hline ITEM & CLINICAL SAMPLE & TOTAL SAMPLE \\
\hline Q1 & 0.760 & 0.870 \\
Q6 & 0.638 & 0.710 \\
Q8 & 0.702 & 0.811 \\
Q10 & 0.672 & 0.752 \\
Q11 & 0.516 & 0.763 \\
\hline
\end{tabular}

a one-factor solution for the clinical sample that explained $27.07 \%$ of variance. Similarly, a onefactor solution was found for the total sample (Kaiser-Meyer-Olkin measure $=0.929$, Bartlett's Test of Sphericity, $\chi^{2}=2356.326, \mathrm{p}<0.001$ ) that explained $42.06 \%$ of variance. Factor loadings are shown in Table 2. For the GAI-SF, the factor analysis for both clinical (Kaiser-Meyer-Olkin measure $=0.753$, Bartlett's Test of Sphericity, $\chi^{2}$ $=133.320, \mathrm{p}<0.001)$ and total samples (KaiserMeyer-Olkin measure $=0.845$, Bartlett's Test of Sphericity, $\chi^{2}=453.275, \mathrm{p}<0.001$ ) suggested a one-factor solution that explained $43.90 \%$ and $61.28 \%$ of variance in clinical and total samples respectively. Factor loadings are shown in Table 3.

Finally, Receiver Operating Characteristic (ROC) analyses were conducted in STATA version 12 to determine the sensitivity and specificity of GAI and GAI-SF in correctly identifying the clinical sample from the non-clinical sample. Test's accuracy is determined by the area under the ROC curve (AUC), where an area of 1 indicates perfect accuracy and 0.5 suggests no greater than chance (see Figure 1). For GAI, the AUC $=0.981$ ( $\mathrm{SE}=$ $0.007, \mathrm{p}<0.001,95 \% \mathrm{CI}=0.967-0.995$. The recommended cutoff of $8 / 9$ had $69.5 \%$ sensitivity, $100 \%$ specificity, and $\kappa=0.513, \mathrm{p}<0.001$. For GAI-SF, the AUC $=0.954(\mathrm{SE}=0.01, \mathrm{p}<0.001$, $95 \% \mathrm{CI}=0.928-0.980)$. The recommended cutoff of $2 / 3$ had a sensitivity $=78.14 \%$, specificity $=$ $98.3 \%, \kappa=0.612 ; \mathrm{p}<0.001$. There was a significant difference between GAI and GAI-SF $\left(\chi^{2}(1)=9.52, p=0.002\right)$, indicating that the full GAI was significantly better at identifying those in the clinical group.

\section{Discussion}

This study aimed to examine the psychometric properties of GAI and GAI-SF in clinical and non-clinical samples. We examined the factor structure, convergent and divergent validity as well and the internal reliability of the short form in comparison with the full version. We also investigated the sensitivity and specificity of the recommended optimal cutoffs for GAI and GAISF to identify those with a diagnosis of anxiety disorder in a psychogeriatric sample with comorbid anxiety and depression. Focusing on GAI first, our findings support previous studies in terms of good internal consistency for GAI, similar to Pachana et al. (2007) and Byrne et al. (2010), and good convergent validity between GAI and PSWQ-A among all samples, similar to Diefenbach et al. (2009). Consistent with previous studies, scores on GAI were significantly related to clinical status. These findings are consistent with previous work suggesting good convergent validity with other selfreport measures of anxiety (Pachana et al., 2007; Byrne et al., 2008; Diefenbach et al., 2009).

For GAI-SF, the internal consistency was adequate in total, non-clinical, and clinical samples, similar to previous findings (Gerolimatos et al., 2013), in a sample of nursing home residents $(\alpha=0.73)$. Convergent validity with self-report measures was good for total and non-clinical samples, consistent with the development paper (Byrne and Pachana, 2011). A one-factor solution was suggested for both GAI and GAI-SF.

Similar to previous studies, GAI and GAISF were significantly correlated with GDS in all samples. While previous studies have been inconsistent with their reporting of this relationship as either evidence of good convergent validity (given the high rates of comorbidity in late-life anxiety and depression) or poor divergent validity (given the poor differentiation), we would suggest that this is an evidence of poor divergent validity. 


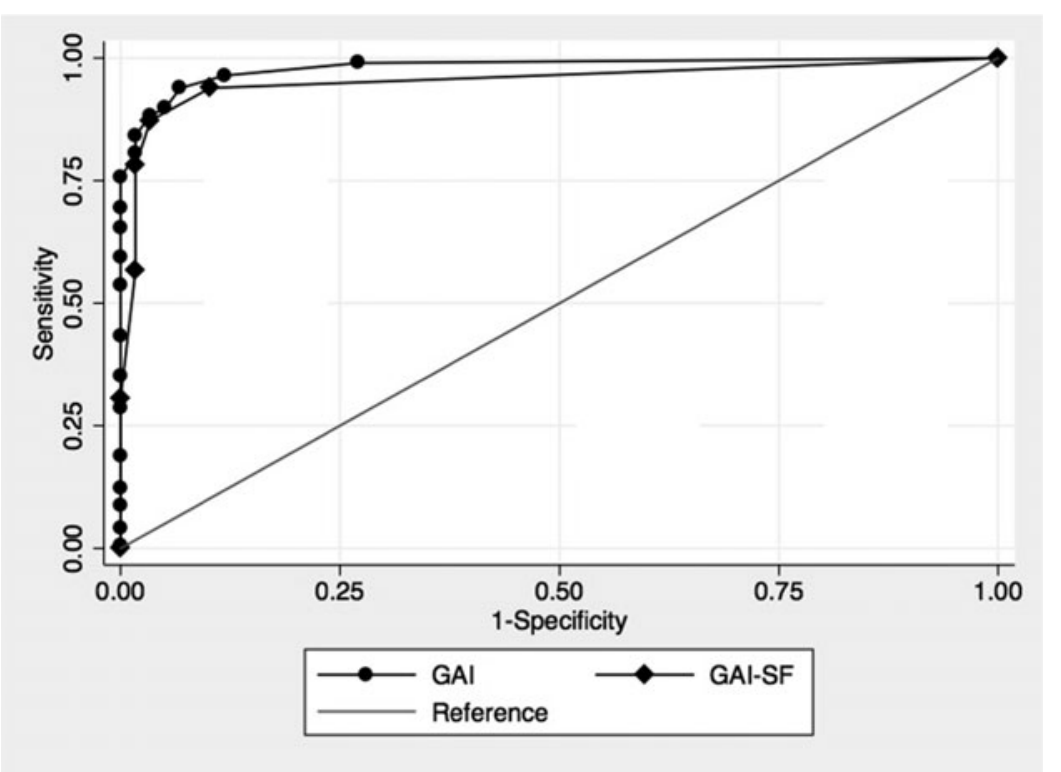

Figure 1. ROC analysis for GAI and GAI-SF using clinical and non-clinical samples

Note: GAI = Geriatric Anxiety Inventory; GAI-SF = Geriatric Anxiety Inventory - Short Form; ROC = Receiver Operating Characteristics.

The correlation between GAI and GDS in the clinical sample was significantly weaker than the relationship between GAI and PSWQ-A, suggesting adequate divergent validity in the clinical sample. However, the magnitude of correlations was similar in total and non-clinical samples, suggesting poorer divergent validity from depressive symptoms in total and non-clinical samples. The GAI-SF experienced similar issues with GAI for divergent validity: better divergent validity in the clinical sample, and poorer divergent validity from depression in total and non-clinical samples. The poor divergent validity from GDS in the non-clinical sample is likely a consequence of overlap of anxiety and depression construct more generally, and has been shown to be a common problem with other measures of anxiety, for example, the PSWQ (Hopko et al, 2003). Divergent validity from measures of cognitive functioning was good in all the samples.

Both GAI and GAI-SF demonstrated good predictive validity for clinical status; however, the ROC analysis suggested that the full GAI was significantly better at identifying those in the clinical group. Previous studies examining cutoff scores have used very small numbers of patients with a diagnosed anxiety disorder (Pachana et al., 2007; Diefenbach et al., 2009; Byrne et al., 2010; Cheung et al., 2012; Matheson et al., 2012). This is the first study to include a large sample of older adults with a diagnosed anxiety disorder, but replicates previous findings. The ROC analysis suggested adequate sensitivity and specificity using the recommended cutoff of $8 / 9$ for GAI for identifying people with an anxiety disorder, resulting in a sensitivity of $69.5 \%$ and specificity of $100 \%$. Similarly, the recommended cutoff of $2 / 3$ for GAI-SF was adequate with $78.14 \%$ sensitivity and $98.3 \%$ specificity. This study adds to the emerging body of evidence supporting the utility of GAI-SF as a valid screening measure for anxiety disorders in older adults.

We acknowledge potential limitations of this study, including that all clinical participants had a comorbid depressive disorder diagnosis that may have escalated the divergent validity relationship with depression measures. However, given that previous studies have found a significant relationship with measures of depression, and that the weakest relationship was among the clinical sample, we would suggest that the comorbidity of our sample did not unduly influence results. Similarly, the nonclinical sample in this study reported minimal levels of anxiety, with only $10.2 \%$ of the sample participants endorsing any anxiety symptoms on GAI-SF and $27.1 \%$ endorsing any symptoms on GAI. This may have created a floor effect for ROC analyses, whereby endorsing almost any symptoms on GAI would likely indicate clinical status. Although the present sample ranged in age from 60 to 88 years, the mean age for the total sample indicated that it was a relatively young older adult sample and the results warrant replication in an older sample. A final limitation is that we extracted the GAISF scores from the full GAI, as done in Byrne and Pachana (2011). While this reduces potential error caused by administering the scale twice, it is important that further comparisons are made when the two scales are administered separately. 
This study is one of the first to examine the psychometric properties of GAI and GAI-SF in a large clinical sample. Results support the existing research that GAI is adequate for detecting anxiety in community-dwelling clinically disordered and non-clinical older adults. Further, our results indicated that GAI-SF is also an adequate measure of anxiety. Although the ROC results indicated that GAI is more accurate at identifying clinical status than the short version, the short version may be useful for screening purposes in primary care or epidemiological settings. Replication of these findings in independent studies using large numbers of clinical participants is needed.

\section{Conflict of interest}

None

\section{Description of authors' roles}

C. Johnco and V. Wuthrich designed the study, collected and analyzed the data, and wrote the manuscript. A. Knight was responsible for assisting with the statistical analysis of the paper and manuscript preparation. D. Tadic was responsible for collecting the data and revising the paper.

\section{References}

Andrew, D. and Dulin, P. (2007). The relationship between self-reported health and mental health problems among older adults in New Zealand: experiential avoidance as a moderator. Aging and Mental Health, 11, 596-603. doi:10.1080/13607860601086587.

Beck, A. T., Epstein, N., Brown, G. and Steer, R. A. (1988). An inventory for measuring clinical anxiety: psychometric properties. Fournal of Consulting and Clinical Psychology, 56, 893-897. doi:http://dx.doi.org/10.1037/ 0022-006X.56.6.893.

Boddice, G., Pachana, N. A. and Byrne, G. J. (2008). The clinical utility of the geriatric anxiety inventory in older adults with cognitive impairment. Nursing Older People, 20, 36-39.

Byrne, G. J. and Pachana, N. A. (2011). Development and validation of a short form of the Geriatric Anxiety Inventory - The GAI-SF. International Psychogeriatrics, 23, 125-131. doi:10.1017/S1041610210001237.

Byrne, G. J., Pachana, N. A., Arnold, L., Chalk, J. B. and Appadurai, K. (2008). P2-239: performance characteristics of the geriatric anxiety inventory in memory clinic attendees. Alzheimer's and Dementia, 4, T441-T442. doi:10.1016/j.jalz.2008.05.1314.

Byrne, G. J., Pachana, N. A., Goncalves, D. C., Arnold, E., King, R. and Khoo, S. K. (2010). Psychometric properties and health correlates of the Geriatric Anxiety
Inventory in Australian community-residing older women. Aging and Mental Health, 14, 247-254. doi:10.1080/13607861003587628.

Carlson, M. et al. (2011). Psychometric properties of reverse-scored items on the CES-D in a sample of ethnically diverse older adults. Psychological Assessment, 23, 558-562. doi:10.1037/a0022484.

Cheung, G. (2007). Concurrent validity of the Geriatric Anxiety Inventory in late-life depression. International Psychogeriatrics, 19, 333-335. doi:doi:10.1017/S1041610206004340.

Cheung, G., Patrick, C., Sullivan, G., Cooray, M. and Chang, C. L. (2012). Sensitivity and specificity of the Geriatric Anxiety Inventory and the Hospital Anxiety and Depression Scale in the detection of anxiety disorders in older people with chronic obstructive pulmonary disease. International Psychogeriatrics, 24, 128-136. doi:10.1017/S1041610211001426.

Di Nardo, P. A., Brown, T. A. and Barlow, D. H. (1994). Anxiety Disorders Interview Schedule for DSM-IV. Boston, MA: Center for Stress and Anxiety Related Disorders, Boston University.

Diefenbach, G. J., Tolin, D. F., Meunier, S. A. and Gilliam, C. M. (2009). Assessment of anxiety in older home care recipients. The Gerontologist, 49, 141-153. doi:10.1093/geront/gnp019.

Feldman, L. A. (1993). Distinguishing depression and anxiety in self-report: evidence from confirmatory factor analysis on nonclinical and clinical samples. Fournal of Consulting and Clinical Psychology, 61, 631-638. doi:http://dx.doi.org/10.1037/0022-006X.61.4.631.

Gerolimatos, L. A., Gregg, J. J. and Edelstein, B. A. (2013). Assessment of anxiety in long-term care: examination of the Geriatric Anxiety Inventory (GAI) and its short form. International Psychogeriatrics, 25, 1533-1542. doi: $10.1017 /$ S1041610213000847.

Goldberg, D., Bridges, K., Duncan-Jones, P. and Grayson, D. (1988). Detecting anxiety and depression in general medical settings. British Medical fournal, 297, 897-899. doi:http://www.ncbi.nlm.nih.gov/pmc/articles/ PMC1834427/pdf/bmj00306-0033.pdf.

Green, J., Goldstein, F., Sirockman, B. E. and Green, R. C. (1993). Variable awareness of deficits in Alzheimer's disease. Neuropsychiatry, Neuropsychology, and Behavioral Neurology, 6, 159-165.

Hazlett-Stevens, H., Ullman, J. B. and Craske, M. G. (2004). Factor structure of the Penn State Worry Questionnaire: examination of a method factor. Assessment, 11, 361-370. doi:10.1177/1073191104269872.

Hopko, D. R. et al. (2003). Assessing worry in older adults: confirmatory factor analysis of the Penn State Worry Questionnaire and psychometric properties of an abbreviated model. Psychological Assessment, 15, 173-183. doi:http://dx.doi.org/10.1037/1040-3590.15./?PMU ?) 2.173

Johnco, C., Wuthrich, V. M. and Rapee, R. M. (2013). The role of cognitive flexibility in cognitive restructuring skill acquisition among older adults. Fournal of Anxiety Disorders, 27, 576-584. doi:10.1016/j.janxdis.2012.10.004.

Jongenelis, K. et al. (2005). Diagnostic accuracy of the original 30-Item and shortened versions of the Geriatric Depression Scale in nursing home patients. International 
Fournal of Geriatric Psychiatry, 20, 1067-1074. doi:http://dx.doi.org/10.1002/gps.1398.

Kieffer, K. M. and Reese, R. J. (2002). A reliability generalization study of the Geriatric Depression Scale. Educational and Psychological Measurement, 62, 969-994. doi:http://dx.doi.org/10.1177/0013164402238085.

Matheson, S. F. et al. (2012). Validity and reliability of the geriatric anxiety inventory in Parkinson's disease. Australasian fournal on Ageing, 31, 13-16. doi:10.1111/j.1741-6612.2010.00487.x.

Meyer, T. J., Miller, M. L., Metzger, R. L. and Borkovec, T. D. (1990). Development and validation of the Penn State Worry Questionnaire. Behaviour Research and Therapy, 28, 487-495. doi:10.1016/00057967(90)90135-6.

Mioshi, E., Dawson, K., Mitchell, J., Arnold, R. and Hodges, J. R. (2006). The Addenbrooke's Cognitive Examination revised (ACE-R): a brief cognitive test battery for dementia screening. International fournal of Geriatric Psychiatry, 21, 1078-1085. doi:http://dx.doi.org/ 10.1002/gps.1610.

Newman, M. G. et al. (2002). Preliminary reliability and validity of the Generalized Anxiety Disorder Questionnaire-IV: a revised self-report diagnostic measure of generalized anxiety disorder. Behavior Therapy, 33, 215-233. doi:http://dx.doi.org/10.1016/S0005$7894 \% 2802 \% 2980026-0$.
Pachana, N. A., Byrne, G. J., Siddle, H., Koloski, N., Harley, E. and Arnold, E. (2007). Development and validation of the Geriatric Anxiety Inventory. International Psychogeriatrics, 19, 103-114. doi:10.1017/S1041610206003504.

Rozzini, L. et al. (2009). Anxiety symptoms in mild cognitive impairment. International fournal of Geriatric Psychiatry, 24, 300-305. doi:http://dx.doi.org/10.1002/ gps.2106.

Spielberger, C. D., Gorsuch, R. and Lushene, R. E. (1970). Manual for the State-Trait Anxiety Inventory. Palo Alto, CA: Consulting Psychologists Press.

Stulz, N. and Crits-Christoph, P. (2010). Distinguishing anxiety and depression in self-report: purification of the Beck Anxiety Inventory and Beck Depression Inventory-II. fournal of Clinical Psychology, 66, 927-940. doi: $10.1002 /$ jclp. 20701.

Wuthrich, V. M. and Rapee, R. M. (2013). Randomized controlled trial of group cognitive behavioral therapy for comorbid anxiety and depression in older adults. Behaviour Research and Therapy, 51, 779-786. doi: http://dx.doi.org/10.1016/j.brat.2013.09.002.

Yesavage, J. A. et al. (1983). Development and validation of a geriatric depression screening scale: a preliminary report. Fournal of Psychiatric Research, 1, 37-49. doi:http://dx.doi.org/10.1016/0022-3956(82) 90033-4. 